\title{
Cellular factors derived from mesenchymal stem and progenitor cells with regeneration effects
}

\author{
Tsung Dao Lee ${ }^{1}$, Han Zhang ${ }^{1}$, Heying Wang $^{1}$, Lei-Fa Chang ${ }^{2}$ \\ ${ }^{1}$ Department of Research and Development, Sino-American Biotech Co., Ltd., Beijing, 102206, China; \\ ${ }^{2}$ Asia-Med Medical Reference Laboratory, Taipei, Taiwan, China
}

\begin{abstract}
Regeneration effects with cellular factors are considered essential in regenerative treatments. Cellular factors derived from multiple cells can be applied in such therapies. Various clinical trial phases are based on studies of mesenchymal stem and progenitor cells (MSPCs). Mesenchymal stem cells (MSCs) are pluripotent stem cells which have multi-directional differentiation potential. MSPCs may exhibit full stem cell functions and can be obtained from different tissues, such as adipose tissue, umbilical cord and bone marrow etc. MSPCs reside in the perivascular niche that is proximal to blood vessels, which allow MSPCs capable of exerting their potential of homing and migration across the endothelium barrier toward lesion sites for tissue repairing or regeneration. MSPCs can be stimulated to release various factors, including surface molecules, growth factors and inhibitory factors. MSPCs' homing potential depends on the expression of certain surface molecules. The growth and inhibitory factors contribute to tissue regeneration and immunomodulation effects. This review provides details of how cellular factors derived from MSPCs can be used for homing and repair mechanisms, and ultimately be applied to clinical settings.
\end{abstract}

Keywords: regeneration, cellular factors, homing, repair, immunomodulation, MSPCs

\section{INTRODUCTION}

The development of tissue regeneration methods has become increasingly important. Their therapeutic effects depend on cellular factors, which possess the capacity of modulation tissue inflammation and homeostasis, ultimately achieving tissue regeneration. Cellular factors are released by multiple cells such as stem, progenitor or primary cells. Various clinical trial phases are largely based on the study of cellular factors derived from MSPCs. Hence, we conducted this review to cover the basic mechanisms of such therapies via MSPCs derived cellular factors.

In human fertilization, an ovum and a haploid

*Correspondence to: Tsung Dao Lee PhD. Department of Research and Development, Sino-American Biotech Co., Ltd., Beijing, 102206, China.E-mail:johnleea@126.com.

The authors declare no conflicts of interest. sperm cell combine together resulting in a fertilized egg, which creates a single toti-potential cell. Pluripotent stem cells from blastocysts can be isolated from a culture with the use of specialized media to differentiate the cells into different cell lineages such as hematopoietic stem cells, neural stem cells and MSCs. MSCs are characterized morphologically as having a long thin and reticular fibril, and also molecularly by the presence of a stemness DNA signature. MSCs and their immediate progenitor cells are collectively known as MSPCs, can be isolated from Wharton's jelly, and exhibit a mesenchymal-like shape with a flat polygonal morphology ${ }^{[1]}$, which give MSPCs the ability to catch onto the surface of flasks. MSPCs are non-hematopoietic stromal cells derived from mesoderm, have multi-directional differentiation potential, and consequently can differentiate into tissue specific cells such as osteocytes, chondrocytes, adipocytes etc. ${ }^{[2,3]}$. MSCs are identified with low im- 
munogenicity and high immunomodulation properties which results in a low expression of HLA class I and a null expression in HLA class II in general ${ }^{[4]}$. MSCs are defined by variable markers such as positive markers CD73, CD90, CD105 and negative markers CD45, CD34, CD11b, etc. MSCs can be derived from many different tissue sources including bone marrow, umbilical cord and adipose tissue etc. Depending upon the tissue or organ of origin, MSCs may express different positive and negative markers.

MSPCs are traditionally isolated in vitro, based on their plastic adherence properties. However, not all fibroblast-like plastic adherent cells meet the generally accepted criteria of MSCs, including stem cell activity $^{[5]}$. To eliminate confusion and to be more accurate regarding nomenclature, the International Society for Cellular Therapy's (ISCT) official statement encourages the scientific community to use the term "mesenchymal SCs" only for cells that meet specified SC criteria, while those that shouldn't be termed "multipotent mesenchymal stromal cells". In analogy to the terms 'HSPCs', the 'MSPCs' was referred to mesenchymal stem and progenitor cells. The Mesenchymal and Tissue Stem Cell Committee of the ISCT defined MSPCs officially as having the following qualities: (1) Must be plastic-adherent in culture. (2) Must express CD73, CD90 and CD105; must lack expression of CD11b, CD14, CD34, CD45, CD79a, and human leukocyte antigen-D-related (HLA-DR). (3) Must have the capacity to differentiate into osteoblasts, adipocytes and chondroblasts in vitro ${ }^{[5,6]}$.

The heterogeneous populations with different degrees of "stemness" and most MSPCs populations exhibit full SC function, including the capacity for longterm self-renewal. In this article we analyzed MSPCs (as defined by the ISCT) to specify their functions and characteristics ${ }^{[6]}$.

\section{CELLULAR FACTORS RELEASING PROPERTIES}

MSPCs possess cellular factor-releasing properties which play an essential role in regeneration therapy. Inflammatory chemokines ${ }^{[1]}$ released by inflammatory sites recruit $\mathrm{T}$ cells and antigen presenting cells (APCs) toward lesion sites. During an immune response, inflammatory cytokines ${ }^{[2]}$ produced by T cells and APCs modulate the function of MSPCs leading to the secretion of growth factors and inhibitory factors and the expression of certain surface molecules on MSPCs ${ }^{[7,8]}$. The activation of MSPCs by inflammatory cytokines is an inflammatory cytokine licensing process. The licensed MSPCs' homing toward a lesion site is governed by the surface molecules such as VLA-4, CXCR4 that are involved in capture, rolling, firm adhesion and transendothelial migration. The growth factors secreted by MSPCs may promote the repair and regeneration of damaged sites in certain organs/tissues. Usually a specific group of growth factors work in favor of creating a trophic microenvironment surrounding the damaged tissue site for regeneration. MSPCs and the immune system interact closely by MSPCs derived-inhibitory factors such as $\mathrm{PGE}_{2}$ and IDO, which can inhibit NK cells and cytotoxic $\mathrm{T}$ cells, and also modulate regulatory $\mathrm{T}$ cell $\left(\mathrm{T}_{\text {reg }}\right)$ function ${ }^{[9,10]}$. In normal conditions, one single bio-active factor cannot be utilized in one specific function. A group of factors are suggested for a specific function during tissue regeneration. Cellular factors co-work with each other to achieve the eventual regeneration of inflammatory sites. These factors can be classified in particular functions: chemo-attraction, immunomodulation, anti-apoptosis, angiogenesis and anti-fibrosis in general ${ }^{[9]}$. Furthermore, cellular factors can balance the concentration of proteolytic enzymes (matrix metalloprotease, i.e. MMPs) and tissue inhibitor MMPs. MSPCs cellular factors' releasing properties may perfectly explain tissue regeneration effects (Fig.1).

\section{HOMING MECHANISM}

MSPCs possess an intrinsic homing ability to migrate toward injured tissues and actively participate in tissue repair. On the basis of MSPCs, obtained from various tissues and their homing ability, a perivascular niche has been identified. A perivascular niche is situated in extremely proximity to the sinusoid in the extracellular matrix, illustrating the reason why MSPCs are able to be derived from various tissues such as bone marrow and also vascularised sites $^{[11]}$. When tissue damage occurs, MSPCs either in the immediate vicinity of perivascular niche from the lesion site or those derived from the bone marrow are believed to migrate into the damaged tissue. It is suggested that MSPCs migrate in multiple steps to the lesion site through a sequence of events mediated by cellular factors. Firstly, MSPCs activated by inflammatory cytokines tend to be licensed MSPCs and migrate via blood circulation reaching the specific lesion site. Secondly, MSPCs are captured by the endothelium and roll on the endothelium wall, which are primarily mediated by selectins. Thirdly, firm adhesion between MSPCs and the endothelium are mediated by a series of ligand/receptor pairs, especially integrins. Firm ad- 
hesion leads MSPCs to crawl on the endothelium wall and scan for weak points to overcome the endothelium barrier. After searching for exit cues, MSPCs are polarized by certain regulators and overcome the endothelium barrier, basal lamina and pericyte sequentially before continuing inflammatory cytokineguided interstitial migration to eventually enter the lesion site. Previous studies regarding MSPCs homing mechanisms are largely based on MSPCs prepared from exogenous sources. The mechanisms of MSPCs migration still remain unclear. However there is currently no evidence that indicate differences in migration toward a lesion site between endogenous MSPCs and exogenous MSPCs ${ }^{[12]}$ (Fig.2).

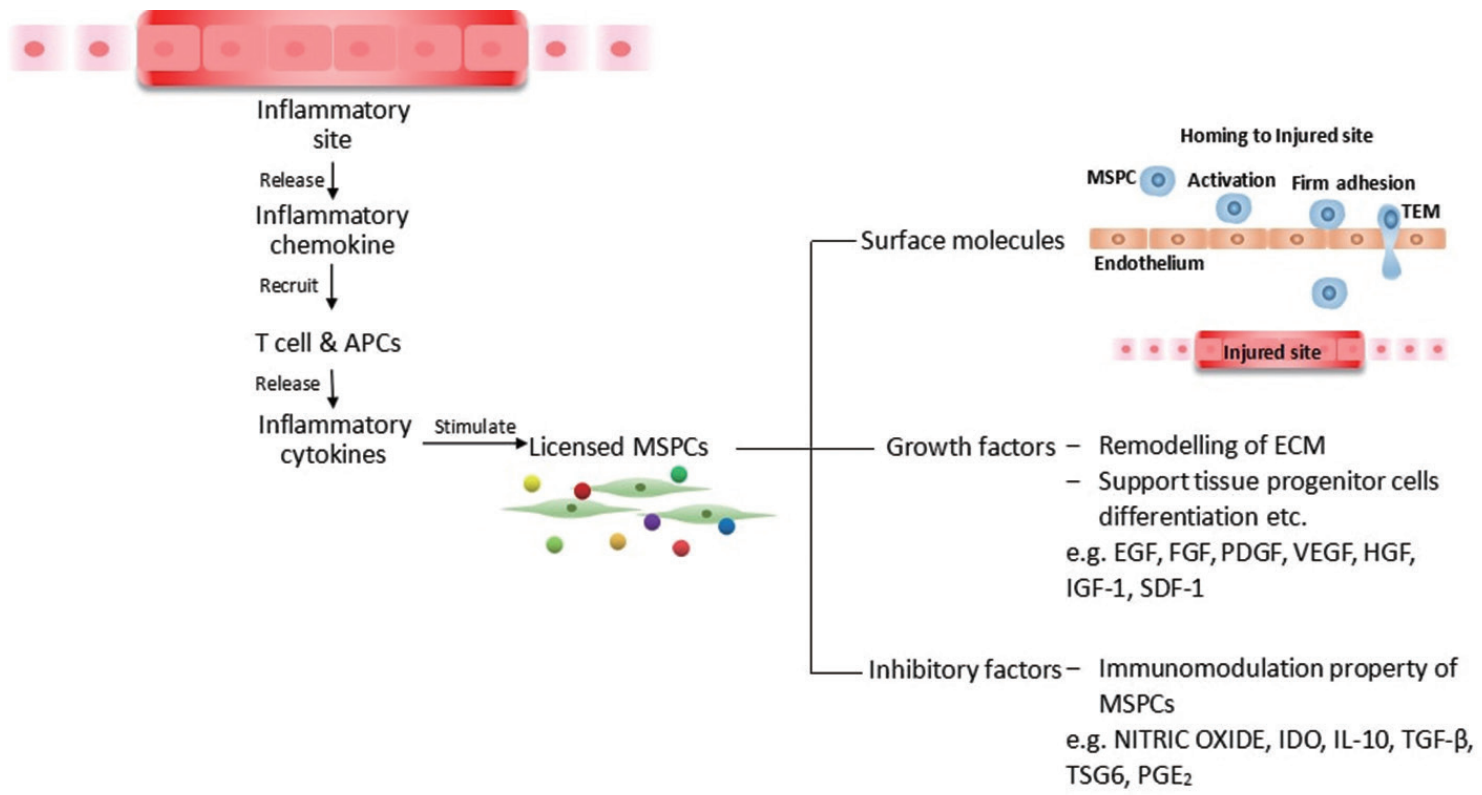

Fig. 1. Under stimulation by inflammatory cytokines, licensed MSPCs secret a spectrum of factors such as surface molecules, growth factors and inhibitory factors. Surface molecules play an essential role in homing of MSPCs toward inflammatory sites. Growth factors may create a trophic microenvironment for tissue regeneration. Inhibitory factors possess immunomodulation property on cells of the immune system.

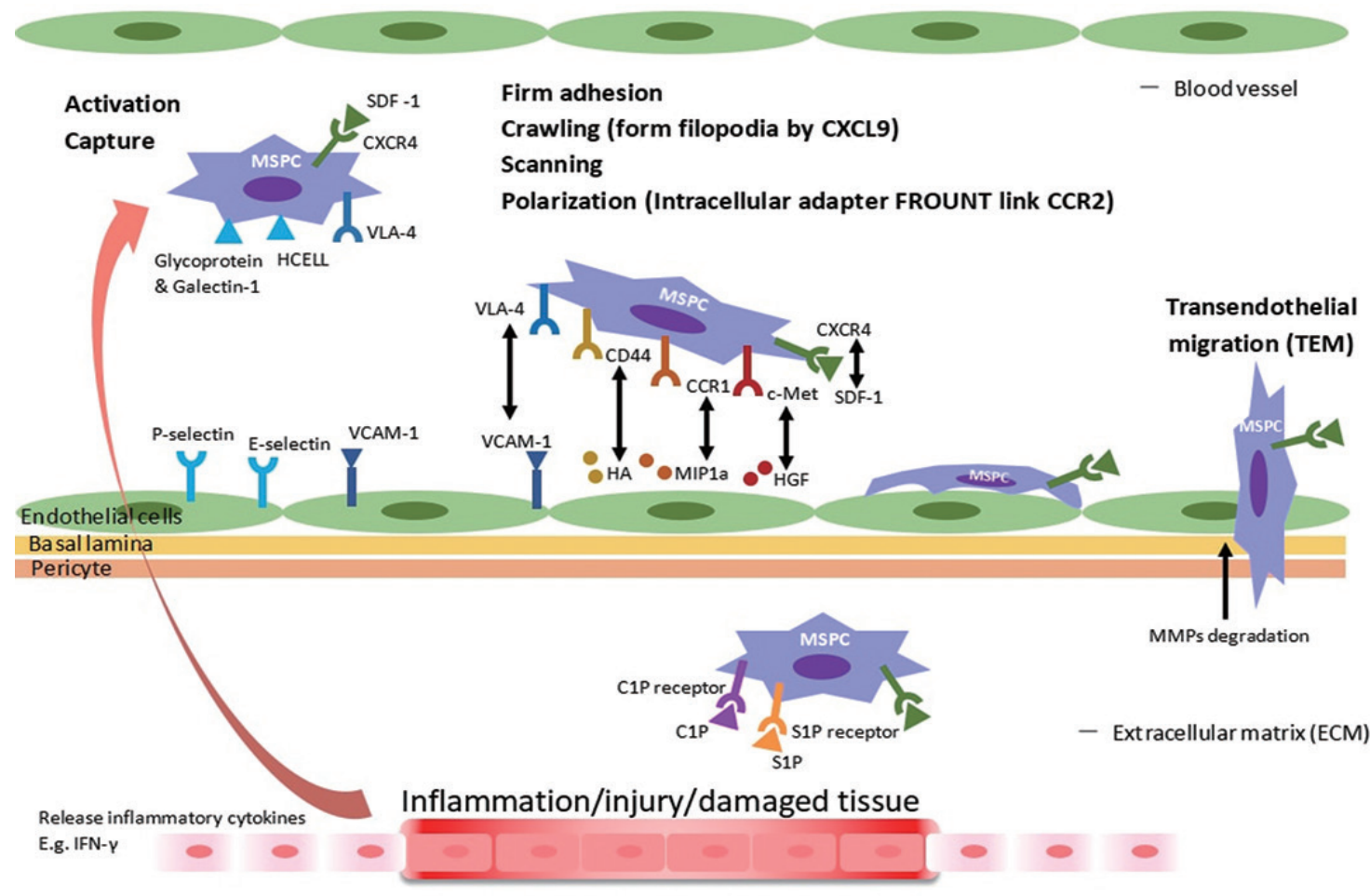

Fig. 2. Homing mechanism of MSPCs toward injured tissue. 


\section{I . Activation}

$\mathrm{T}$ cells and APCs from inflammatory sites release inflammatory cytokines (e.g. IFN $-\gamma, \mathrm{TNF}-\mathrm{a}$, etc.). Inflammatory cytokines act as signals in order to induce and modulate MSPCs function. MSPCs activated by inflammatory cytokines tend to be licensed MSPCs that have the capacity to home to and repair inflammatory tissue. Licensed MSPCs may sense inflammatory sites by the inflammatory cytokine-gradient and express surface molecules on its membrane, preparing for further actions with the endothelium. In response to mediators released by inflamed tissue, endothelial cells are activated by the upregulation of receptors on the endothelium, which contribute to the homing of MSPCs ${ }^{[13]}$. Those mediators such as TNF-a can induce the activation of $\mathrm{P}$-selectins on the endothelial cells surface within a few minutes, increase E-selectins expression and reach peaks after $3-4$ hours ${ }^{[8]}$. Vascular cell adhesion molecule 1 (VCAM-1) expressed by the endothelium is also stimulated by inflammatory cytokines from the activation of intracellular signaling pathways. MSPCs expressed receptor VLA-4 interact with corresponding ligand VCAM-1. Inflammatory cytokines upregulate VCAM-1 and activate VLA-4, leading to the initial arrest of MSPCs on the endothelium surface ${ }^{[12]}$. The chemokine stromal cell-derived factor 1 (SDF1, also known as CXCL12) is critical for MSPCs chemotaxis and homing in response to inflammatory cytokines through interactions with CXCR4 on the MSPCs surface ${ }^{[14]}$.

\section{II . Capture}

Licensed MSPCs are shown to express ligands: galectin-1/glycoprotein and HCELL which bind to corresponding receptors' $\mathrm{P}$-selectins and $\mathrm{E}$-selectins expressed in the endothelium, respectively. These two pairs of ligand/receptors initiate the capture of MSPCs to the endothelium and mediate further rolling on the endothelium, while platelet bridging assists with Pselectins to capture licensed MSPCs ${ }^{[12,14]}$.

\section{III . Rolling}

MSPCs rolling is obligatory and is facilitated by galectin-1 binding with $\mathrm{P}-$ selectins or platelet bridging, which act as alternatives for selectin-mediated adhesion. Besides selectins mediate rolling, integrin VLA-4 is also an important mediator of rolling and arresting MSPCs at the sites of inflammation ${ }^{[13]}$. Due to the possibility of MSPCs being passively arrested or slowed down, the rolling of MSPCs may not be crucial for establishing firm adhesion ${ }^{[12,14]}$.

\section{IV . Firm adhesion}

The activation of surface molecules to mediate firm adhesion between MSPCs and endothelium is a core mechanism of the homing process. The expression of numerous ligands and receptors are shown to contribute for firm adhesion including HA/CD44, MIPla/CCRl, HGF/c-Met, SDF-1/CXCR4 and VCAM1/VLA-4. The key ligand/receptor pair VCAM-1/ VLA-4 is shown in establishing firm adhesion, mediated via Racl/Raql GTPase signaling. HCELL binding to E-selectins may affect VCAM-1/VLA-4 adhesion and triggers activation of $\mathrm{G}$-protein signaling, while bypassing chemokine receptor signaling ${ }^{[12]}$. MIP-la is a chemokine that causes migration of proinflammatory cells to sites of inflammation and regulates their transendothelial migration(TEM). MIPla expression is induced by inflammatory stimuli. MSPCs express CCRl to interact with MIP-la. MIPla may increase the homing of MSPCs to injured sites. However, the chemotaxis of MIP-la still needs to be identified ${ }^{[15,16]}$. Moreover, the concentration of c-Met is upregulated in wound areas and shown to act as a chemoattractant ${ }^{[3]}$ for MSPCs ${ }^{[14]}$.

\section{V . Crawling \& scanning}

Firm adhesion results in licensed MSPCs crawling on the endothelium and form filopodia from the stimulation of CXCL9 (CXCL9 being a CXC-motif, $\mathrm{T}$-cell chemoattractant, which is induced by the inflammatory cytokine-IFN- $\gamma$ ). During crawling, MSPCs scan the endothelium searching for exit cues to overcome the endothelial barrier ${ }^{[12]}$. During in vitro studies on transmigrating MSPCs, blebs are suggested to mediate inter-cellular forces against the endothelium. Also MSPCs and the endothelium actively cooperate to enable guidance by trans-migratory cups, a form of endothelial protrusion ${ }^{[12]}$.

\section{VI . Polarization}

MSPCs require polarization before transendothelial migration and show little lateral migration on the endothelium during polarization. The intracellular adapter molecule FROUNT links to CCR2, followed by CCR2 clustering, which leads to cytoskeletal reorganization ${ }^{[17]}$. Cytoskeletal remodeling facilitates the opening of endothelial junctions and eases the passage of the transmigrating cells ${ }^{[17]}$.

\section{VII . Transmigration}

Generally, during transendothelial migration, MSPCs have to overcome three barriers: the endothelium, basement membrane (basal lamina) and the 
pericyte sheath.

\section{Overcome endothelium}

MSPCs advance through the endothelial barrier by the formation of bleb-like protrusions (cup-like structures) on the cell surface, particularly at sites of close contact with the endothelium. MSPCs overcome the endothelial monolayer also by cooperating with endothelial cells. A few signaling pathways relating to TEM have been identified so far. For example, phosphokinase $\mathrm{C}$ (PKC) is upregulated in an IL-8 dependent manner in human MSPCs. MSPCs TEM is dependent on signaling pathways such as phosphoinositide 3-kinase (PI3K)/Akt and Rho kinase (ROCK). As integrins lack enzymatic activity, an intracellular adapter transduces signals from outside or from the inside, one intracellular adapter integrin-linked kinase (ILK) plays a role for MSPCs TEM $^{[12]}$.

\section{Overcome basal lamina}

Basal lamina is consisted of collagen, lamin and fibronectin in general. As soon as the leading edge reaches the basal lamina, the MMPs are synthesized and released under inflammatory cytokines' stimulation. Pro-inflammatory cytokines: IFN $-\gamma$ and TNF- $a$ increase production of MMPs in MSPCs, thereby enhancing the capacity of MSPCs to migrate through the extracellular matrix ${ }^{[18,19]}$. MMPs are able to degrade basal lamina components; collagen type IV and tissue inhibitor of metalloprotease 3 (TIMP-3). Urokinase-type plasminogen activator (PA) found in MSPC-protrusions can support the migration of MSPCs via the ERK1, 2/MAPK signaling ${ }^{[12]}$.

\section{Overcome pericyte}

Pericytes consist of a contractile cell layer that wrap around endothelial cells. ICAM-1 interplay regulates the passage through the pericyte sheath ${ }^{[12]}$.

Due to different conditions of injury sites, the chemo-attractants may vary. Ceramide-1-phosphate (CIP) and sphingosine-1 phosphate (SIP) are bioactive lipids that resist degrading enzymes. SDF-1 may be degraded by proteolytic enzymes surrounding inflammatory sites. CIP and SIP bind with their corresponding receptors which are subsequently expressed by MSPCs as chemotactic homing signals. Complement is the major humoral component of the innate immune response. The complement cascade activated by different pathways leads to the generation of bioactive peptides such as $\mathrm{C} 3 \mathrm{a}$ and $\mathrm{C} 5 \mathrm{a}$, which possess chemo-attractant activity on MSPCs ${ }^{[14,20]}$.

\section{REPAIR MECHANISM}

While tissue is being injured or damaged, MSPCs and cells of the immune system migrate from blood vessels across extracellular matrix $(\mathrm{ECM})$ to reach the injured tissue by the stimulation of inflammatory cytokines. Since MSPCs have multi-directional differentiation potential, it is believed that MSPCs can differentiate into functional cells to replace the damaged cells. Meanwhile, in response to inflammatory cytokines, MSPCs release a broad spectrum of factors for tissue regeneration. A large amount of growth factors (including transcription factors) are produced to either stimulate MSPCs into endothelial progenitor cells, fibroblasts and tissue progenitor cells or to directly facilitate the progenitor cells differentiation ${ }^{[8,21]}$. The concerted action of growth factors facilitates tissue repair through angiogenesis, remodeling of the extracellular matrix, proliferation and differentiation of tissue progenitor cells for replacement ${ }^{[22]}$. The data reported that growth factors such as HGF and VEGF, seem to improve cell proliferation, inhibit cell apoptosis, and promote cell function via the activation of Akt signaling ${ }^{[21]}$. In addition, evidence points to growth factors such as IGF-1, EGF and BMP-7 etc. being capable of protecting the kidney against fibrosis and restoring its function. BMP-7 is able to improve renal function and regenerate tubular cells ${ }^{[21]}$. Hence, cellular factors derived from MSPCs not only facilitate tissue functional cell differentiation, but also protect the target cells' normal function and work on restoring their function. The inflammatory milieu, including proinflammatory cytokines, strongly inhibits MSPCs differentiation potential. Therefore a sufficient suppression of inflammation prior to an operation is important for efficient tissue regeneration ${ }^{[23]}$. Inhibitory factors modulate the progression of inflammation by modulating immune cells to cease releasing pro-inflammatory cytokines. Depending on the types of immune responses (acute or chronic inflammation), MSPCs may either attenuate the inflammatory response, which leads to the repair of the damaged tissue, or maintain a persistent chronic inflammatory response, leading to fibrosis and deformation of tissue architecture. During acute inflammation, MSPCs (in response to inflammatory cytokines produced by $\mathrm{T}$ cells and APCs) release both growth factors and inhibitory factors. Growth factors promote MSPCs differentiation into functional cells, such as endothelial cells and fibroblasts. The inhibitory factors secreted by MSPCs inhibit immune system cells from releasing pro-inflammatory cytokines. During chronic inflammation, the inhibitory factors are not sufficient to inhibit immune system cells. The growth 
factors continue to assist MSPCs with differentiation into functional cells under stimulation through the low level release of inflammatory cytokines ${ }^{[8,24]}$. Therefore, whether in chronic inflammation or acute inflammation remission, MSPCs are able to release both a low level of growth and inhibitory factors ${ }^{[13]}$. Basically, MSPCs-based therapy depends on two characteristics of MSPCs: MSPCs multi-directional differentiation potential and cellular factors releasing properties (Fig.3).

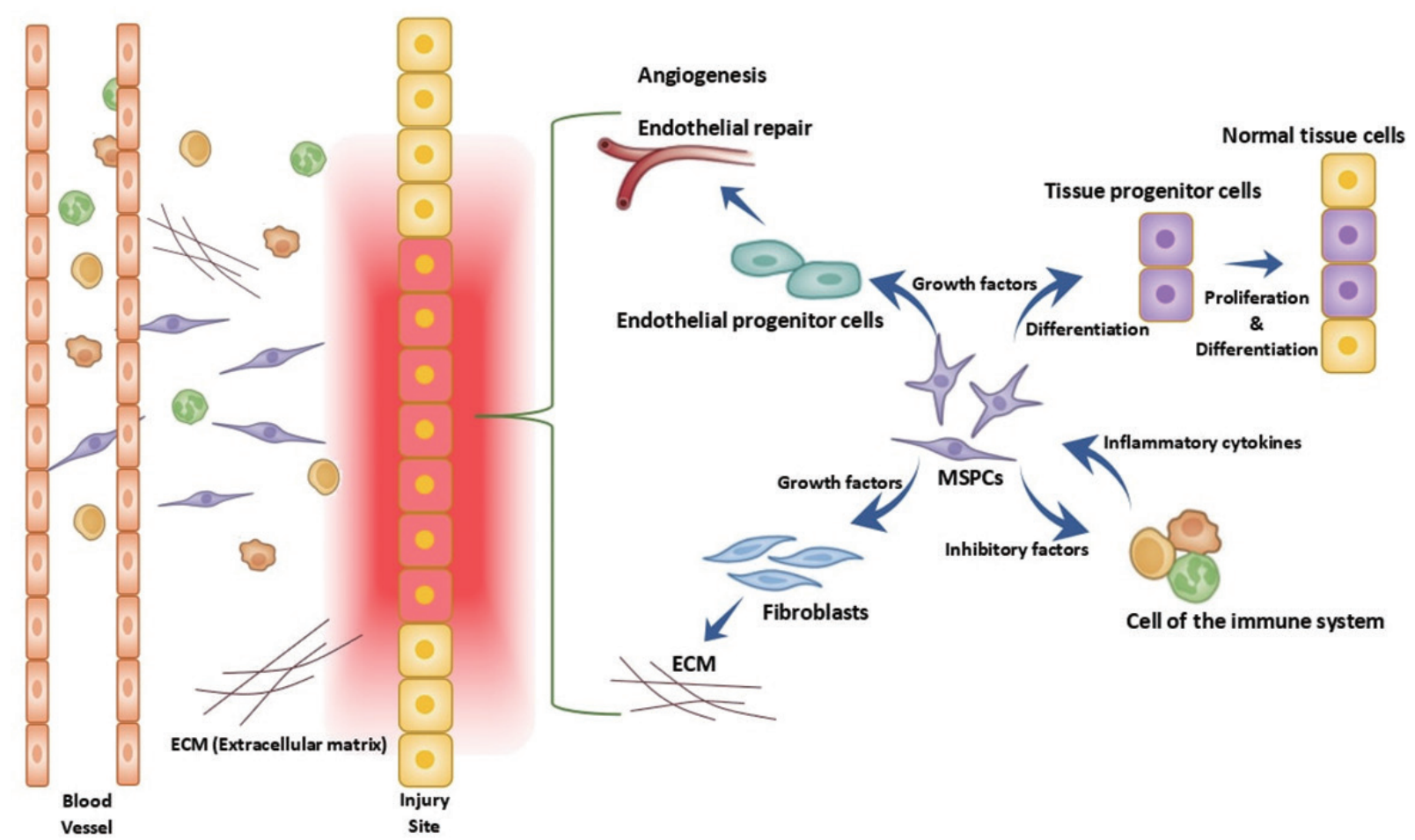

Fig. 3 Repairing mechanism of MSPCs. Under stimulation, licensed MSPCs and cells of the immune system are mobilized toward injury site to exert their functions.

\section{IMMUNOMODULATION PROPERTIES}

Published studies have shown that the immunomodulation ability of MSPCs is not constitutive, instead, it is licensed by inflammatory cytokines in an inflammatory microenvironment ${ }^{[25,26]}$. MSPCs derived cellular factors affect both innate immunity and adaptive immunity. Regarding innate immunity, various factors influence macrophages and NK cells. Macrophages are key effector cells in innate immunity and are involved in tissue defense, homeostasis and reparation ${ }^{[26]}$. They can exhibit either pro-inflammatory or anti-inflammatory phenotype depending on the microenvironment associated with the successive phases of the inflammatory response. MSPCs are able to polarize pro-inflammatory M1 macrophages into anti-inflammatory M2 phenotypes through $\mathrm{PGE}_{2}$ interaction with EP2 and EP4 receptors on macrophages to induce the secretion of IL-10 ${ }^{[27,28]}$. Moreover, MSPCs secret TNF-astimulated gene-6 protein (TSG-6) which interacts through CD44 receptor on resident macrophages. The
CD44 molecule is then dissociated from TLR-2 leading to the impairment of pro-inflammatory cytokine expression. NK cells play a critical role in the defense against virus-infected cells and tumor cells. MSPCs derived factors can modulate NK cells' pro-inflammatory cytokine production. MSPCs inhibit IFN $-\gamma$ secretion by IL-2 or IL-15-activated NK cells. In addition, MSPCs impair NK cells' cytotoxic activity through cell-to-cell contact and soluble factors such as IL-10, PGE $_{2}$, HLA-G5 and TGF- a to downregulate the natural cytotoxicity receptors, NKp30 and NKp44, and the NK group 2D (NKG2D) ${ }^{[26,29]}$. For adaptive immunity, MSPCs interact with T cells, $\mathrm{B}$ cells and dendritic cells respectively. T cells are the major cellular effectors of adaptive immune response and play a central role in cellular mediated immunity ${ }^{[30]}$. T cell activation is dependent on the binding of antigenpresenting MHC class I and non-antigen-specific co-stimulatory molecules including CD40, CD80 and CD86 expressed by donor cells to their ligands on $\mathrm{T}$ cells. There are three types of $\mathrm{T}$ cell, cytotoxic $\mathrm{CD}^{+} \mathrm{T}^{+}$cells, $\mathrm{CD} 4^{+}$helper $\mathrm{T}$ cells and regulatory $\mathrm{T}$ 
cells $^{[31]}$. All of them are implicated in immune responses in general. $\mathrm{CD}^{+}$cells are cytotoxic activated $\mathrm{T}$ lymphocytes. $\mathrm{CD}^{+} \mathrm{T}$ cells can induce the killing of target cells. $\mathrm{CD}^{+} \mathrm{T}$ cells cytotxcity can be inhibited by HLA-G5 secreted by MSPCs. CD $4^{+} \mathrm{T}$ cells are helper $\mathrm{T}$ cells that can be induced by different stimuli to differentiate into Th1, Th2 and Th17 or $\mathrm{T}_{\text {reg }}$ cells. MSPCs can support inhibition of Thl response, promote the secretion of IL-4 and the T cells phenotype shift to the anti-inflammatory Th2 phenotype. Th17 cells secret not only IL-17 but also IL-17F, IL-21 and IL-2 ${ }^{[25,32]}$. These cytokines most likely cooperate to induce tissue inflammation and Thl7-driven effector functions may be different in different tissues ${ }^{[32]}$. $\mathrm{T}_{\text {reg }}$ comprise of several specialized subsets of $\mathrm{T}$ cells that are able to control immune responses and promote and maintain immune tolerance in an antigen-specific way ${ }^{[16]}$. MSPCs secret soluble factors such as $\mathrm{PGE}_{2}$, TGF- $\alpha$ and HO-1 to induce three major $\mathrm{T}_{\text {reg }}$ cell subsets corresponding to IL- $10^{+} \mathrm{T}$ regulatory $1(\mathrm{Tr} 1)$, TGF- $\mathrm{a}^{+}$T helper 3(Th3) and CD25 ${ }^{+} \mathrm{FOXP}^{+}$natural $\mathrm{T}_{\text {reg }}$-like CD4 ${ }^{+}$cells. These $\mathrm{T}_{\text {reg }}$ cells are functional and can efficiently suppress $\mathrm{T}$-cell proliferation triggered by antigenic peptides ${ }^{[31,33]}$. MSPCs also induce more anti-inflammatory $\mathrm{T}_{\text {reg }}$ cells and promote $\mathrm{T}_{\text {reg }}$ cell proliferation or regeneration. MSPCs can support the inhibition of Thl response by decreasing pro-inflammatory cytokines (TNF-a, IFN- $\gamma$ ) and increasing IL-4. MSPCs also inhibit the differentiation of naive $\mathrm{T}$ cells into Th17 cells by inducing the production of anti-inflammatory cytokines IL-10 and modulating transcription factors through cell-to-cell contact and soluble factors: $\mathrm{PGE}_{2}$ and IL-10 ${ }^{[28,29]}$. Th17 and Thl cells are both pro-inflammatory phenotypes play- ing important roles in inflammatory responses of the human body and have been shown to be involved in various autoimmune diseases ${ }^{[25,29]}$. MSPCs polarize $\mathrm{CD} 4^{+} \mathrm{T}$ cells into $\mathrm{T}_{\text {reg }}$ cells and $\mathrm{Th} 2$ cells in a greater anti-inflammatory phenotype ${ }^{[28,29]}$. B cells play a major role in humoral-mediated immunity. B cells differentiate into immunoglobulin-secreting plasmablasts after antigenic stimulation and are potent APCs. MSPCs increase B cells viability while also inhibiting proliferation after polyclonal stimulation, mimicking the three signals (B cell receptor engagement, costimulation and cytokine or toll-like receptor activation of B cell activation) ${ }^{[34]}$. MSPCs also inhibit $\mathrm{B}$ cell differentiation by decreasing $\mathrm{CD} 38^{+} / \mathrm{CD} 138^{+}$ expression after exposure to dendritic cells, and/or impaired antibody production. MSPCs through cellto-cell contact may also inhibit B cell proliferation and terminal differentiation into plasma cells through the down-regulation of transcription factors. Regulatory B cells $\left(\mathrm{B}_{\text {reg }}\right)$ are a peculiar subset of $\mathrm{B}$ cells that can produce anti-inflammatory cytokine(IL-10) ${ }^{[34]}$. MSPCs increase the percentage of $\mathrm{B}_{\mathrm{reg}} \mathrm{s}$ secreting IL-10. Dendritic cells (DCs) are the most potent APCs that can initiate and regulate adaptive immune responses by promoting antigen-specific T cell activation. MSPCs strongly inhibit the initial differentiation of both CD34 ${ }^{+}$ cells and monocytes into DCs. DCs generated in the presence of MSPCs fail to express pro-inflammatory cytokines (such as TNF- $\mathrm{a}$ and IL-12), class- II MHC and costimulatory molecules, but secret large amounts of anti-inflammatory cytokine IL- $10^{[29,35]}$. It is obvious that cellular factors released by MSPCs are key players during immunomodulation effects, which is beneficial for tissue regeneration (Fig.4).

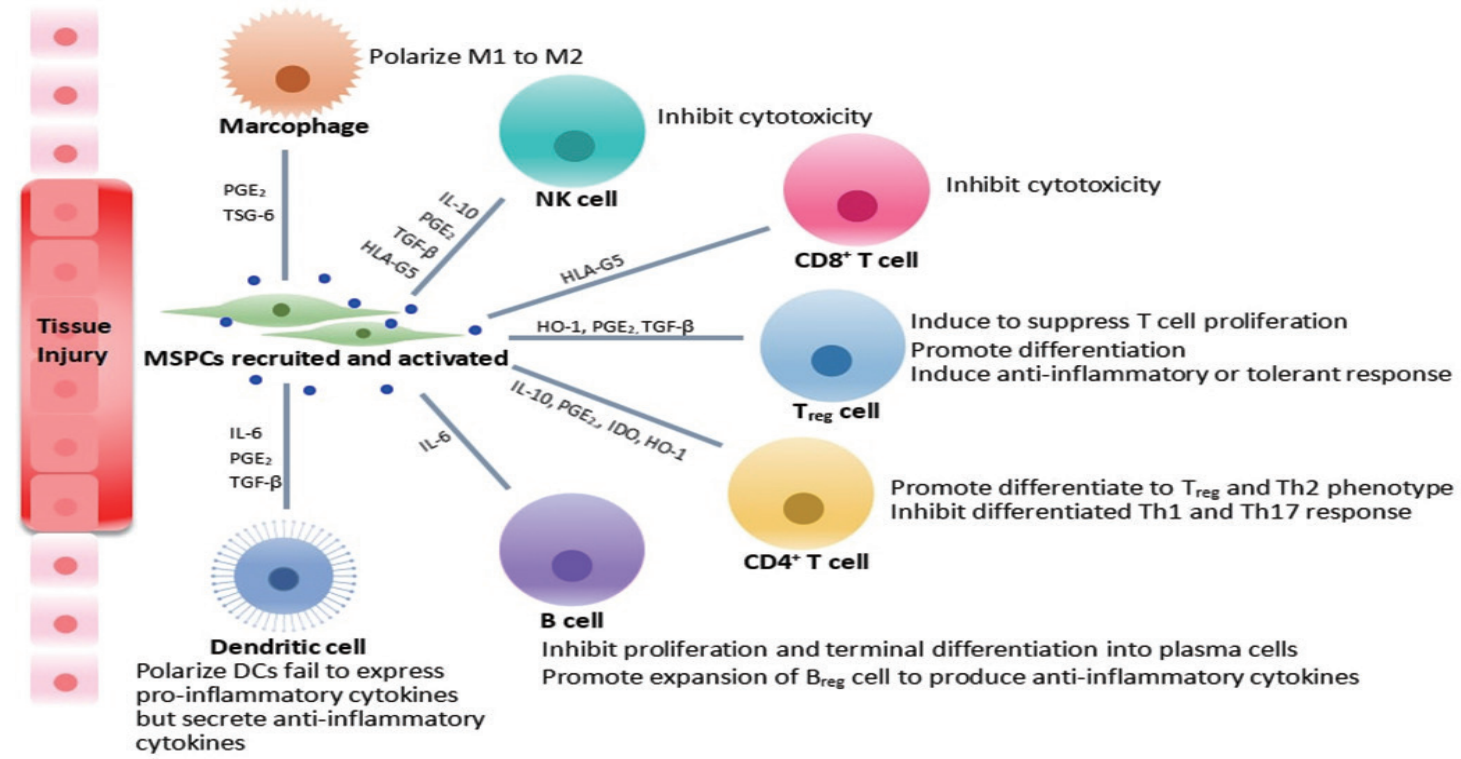

Fig. 4 Immunomodulation properties of MSPCs. MSPCs may exert immunomodulation properties to modulate both innate (macrophages and NK cells) and adaptive immunities (T cells, B cells and DCs). 


\section{CONCLUSIONS}

Cellular factors are promising candidates in regenerative medicine. The benefit of MSPCs is their capability for secreting a broad spectrum of factors in response to inflammatory cytokines. Surface molecules expressed by MSPCs contribute to the homing process, especially in capture, rolling, firm adhesion and TEM between MSPCs and the endothelium. During repair of injured or inflammatory tissue, inhibitory factors are in favor of immunomodulation effects between MSPCs and immune cells. Growth factors released by MSPCs create a trophic microenvironment for endogenous tissue progenitor cell differentiation into normal endogenous tissue cells. This rebuilds the ECM and endothelium surrounding the lesion sites, ultimately achieving anti-inflammation and regeneration at the sites of injured tissue. This flexibility circumvents several difficulties in current standard treatments within clinical applications which will be discussed in succeeding review articles. The distinct advantages of cellular factors over the conventional modalities and other pharmaceuticals may therefore lead to wider applications in the field of regenerative medicine in the future.

\section{ABBREVIATIONS}

\begin{tabular}{ll}
\hline APC & Antigen presenting cell \\
BMP & Bone morphogenetic proteins \\
CCR & C-C chemokine receptor \\
CCL & C-C ligand \\
C1P & Ceramide-1-phosphate \\
CXCL & Chemokine (C-X-C motif) ligand \\
CXCR & Chemokine (C-X-C motif) receptor \\
CTL & Cytotoxic T lymphocyte \\
DC & Dendritic cell \\
EGF & Epidermal growth factor \\
ECM & Extracellular matrix \\
ERK & Extracellular signal-regulated kinases \\
FGF & Fibroblast growth factor \\
HCELL & Hematopoietic cell E-and L-selectin \\
\hline HO-1 & ligand, a specialized glycoform of CD44 \\
HGF & Heme oxygenase 1 \\
HLA & Hepatocyte growth factor \\
HA & Human leukocyte antigen \\
GTPase & Hyaluronic acid \\
IDO & Hydrolyze guanosine-5'-triphosphate \\
IGF & Indoleamine-pyrrole 2, 3-dioxygenase \\
IPC & Insulin growth factor \\
IRS & Insulin producing cell \\
ILK & Insulin receptor substrate \\
IFN & Integrin-linked kinase \\
IL & Interferon \\
FROUNT & Interleukin \\
ICAM-1 & Intracellular adapter \\
\hline
\end{tabular}

\begin{tabular}{ll}
\hline MIP-1 & Macrophage inflammatory protein (CCL3) \\
MHC & Major histocompatibility complex \\
MMP & Matrix metalloprotease \\
MSPC & Mesenchymal stem and progenitor cell \\
MSC & Mesenchymal stem cell \\
MAPK & Mitogen activated protein kinase \\
NK & Natural killer \\
NO & Nitric oxide \\
NKG2D & NK group 2D \\
OPG & Osteoprotegerin \\
PI3K & Phosphoinositide 3-kinase \\
PKC & Phosphokinase C \\
PDGF & Platelet-derived growth factor \\
PGE & Prostaglandin E \\
Treg & Regulatory T cell \\
RA & Rheumatoid arthritis \\
ROCK & Rho kinase \\
S1P & Sphingosine-1 phosphate \\
SDF & Stromal derived factor (CXCL12) \\
Th & T helper cell \\
TIMP & Tissue inhibitor of metalloprotease \\
TLR & Toll like receptor \\
TEM & Transendothelial migration \\
TGF & Transforming growth factor \\
TNF & Tumor necrosis factor \\
TSG & Tumor necrosis factor-inducible gene \\
c-Met & Tyrosine-protein kinase Met \\
PA & Urokinase-type plasminogen activator \\
VCAM & Vascular cell adhesion molecule \\
VEGF & Vascular endothelial growth factor \\
VLA & Very late antigen \\
\hline & \\
\hline
\end{tabular}

\section{References}

[1] Kobolak J, Dinnyes A, Memic A, et al. Mesenchymal stem cells: identification, phenotypic characterization, biological properties and potential for regenerative medicine through biomaterial micro engineering of their niche. Methods, 2016, 15, (99): 62-8.

[2] Kolf CM, Cho E, Tuan RS. Mesenchymal stromal cells. Biology of adult mesenchymal stem cells: regulation of niche, self-renewal and differentiation. Arthritis Res Ther, 2007, 9(1): 204.

[3] Merinogonzález C, Zuñiga FA, Escudero C, et al. Mesenchymal stem cell-derived extracellular vesicles promote angiogenesis: Potential clinical application. Front Physiol, 2016, 9 (7): 24.

[4] Mesenchymal stem cells from extra-embryonic tissues for tissue engineering-Regeneration of the peripheral nerve. In: Gartner A, Pereira T, Gomes R, et al. Editors. Advances in Biomaterials Science and Biomedical Applications, 2013, 10.5772/53336.

[5] Dominici M, Le Blanc K, Mueller I, et al. Minimal criteria for defining multipotent mesenchymal stromal cells. the international society for cellular therapy position statement. Cytotherapy, 2006, 8(4): 315-7.

[6] Pleyer L, Valent P, GreilR. Mesenchymal stem and progenitor cells in normal and dysplastic hematopoiesis' masters of survival and clonality? Int J Mol Sci, 2016, 17(7): 1009. 
[7] Katsuda T, Kosaka N, Takeshita F, et al. The therapeutic potential of mesenchymal stem cell-derived extracellular vesicles. Proteomics, 2013, 13(10-11): 1637-53.

[8] Wang Y, Chen X, Cao W, et al. Plasticity of mesenchymal stem cells in immunomodulation: pathological and therapeutic implications. Nat Immunol, 2014, 15(11): 1009-16.

[9] Caplan AI. Adult mesenchymal stem cells for tissue engineering versus regenerative medicine. J Cell Physiol, 2007, 213(2): 341-7.

[10] Haddad NE. Mesenchymal stem cells: Immunology and therapeutic benefits. In: Ghodamrezanzhad A. Editor. Stem Cells in Clinic and Research. InTech, 2011, 10.5772/2193.

[11] Ghaemi SR, Harding FJ, Delalat B, et al. Exploring the mesenchymal stem cell niche using high throughput screening. Biomaterials, 2013, 34(31): 7601-15.

[12] Nitzsche F, Müller C, Lukomska B, et al. Concise review: MSC adhesion cascade-Insights into homing and transendothelial migration. Stem Cells, 2017, 35(6): 1446-60.

[13] Karp JM, LengTeo GS. Mesenchymal stem cell homing: The devil is in the details. Cell Stem Cell, 2009, 6, 4(3): 206-16.

[14] Marquez-Curtis LA, Janowska-Wieczorek A. Enhancing the migration ability of mesenchymal stromal cells by targeting the SDF-1/CXCR4 axis. Biomed Res Int, 2013, 2013: 561098.

[15] Eseonu OI, De Bari C. Homing of mesenchymal stem cells: mechanistic or stochastic? implications for targeted delivery in arthritis. Rheumatology, 2015, 54(2): 210-8.

[16] Ito H. Chemokines in mesenchymal stem cell therapy for bone repair: a novel concept of recruiting mesenchymal stem cells and the possible cell sources. Mod Rheumatol, 2011, 21(2): 113-21.

[17] Belema-Bedada F, Uchida S, Martire A, et al.Efficient homing of multipotent adult mesenchymal stem cells depends on FROUNT-mediated clustering of CCR2. Cell Stem Cell, 2008, 5, 2(6): 566-75.

[18] Lin W, Xu L, Zwingenberger S, et al. Mesenchymal stem cells homing to improve bone healing. J Orthop Translat, 2017, 9(C): 19-27.

[19] De Becker A, Van Hummelen P, Bakkus M, et al. Migration of culture-expanded human mesenchymal stem cells through bone marrow endothelium is regulated by matrix metalloproteinase-2 and tissue inhibitor of metalloproteinase-3. Haematologica, 2007, 92(4): 440-9.

[20] Rutkowski MJ, Sughrue ME, Kane AJ, et al. The complement cascade as a mediator of tissue growth and regeneration. Inflamm Res, 2010, 59(11): 897-905.
[21] D’Souza N, Rossignoli F, Golinelli G, et al. Mesenchy$\mathrm{mal} \mathrm{stem} / \mathrm{stromal}$ cells as a delivery platform in cell and gene therapies. BMC Med, 2015, 13: 186.

[22] MeirellesLda S, Fontes AM, Covas DT, et al. Mechanisms involved in the therapeutic properties of mesenchymal stem cells. Cytokine Growth Factor Rev, 2009, 20(5-6): 419-27.

[23] Kondo M, Yamaoka K, Tanaka Y. Acquiring chondrocyte phenotype from human mesenchymal stem cells under inflammatory conditions. Int J Mol Sci, 2014, 15(11): 21270-85.

[24] Glenn JD, Whartenby KA. Mesenchymal stem cells: Emerging mechanisms of immunomodulation and therapy. World J Stem Cells, 2014, 6(5): 526-39.

[25] Cao W, Cao K, Cao J, et al. Mesenchymal stem cells and adaptive immune responses. Immunol Lett, 2015, 168(2): 147-53.

[26] Le Blanc K, Mouqiakakos D. Multipotent mesenchymal stem cells and the innate immune system. Nat Rev Immunol, 2012, 12(5): 383-96.

[27] Shin TH, Kim HS, Kang TW, et al. Human umbilical cord blood-stem cells direct macrophage polarization and block inflammasome activation to alleviate rheumatoid arthritis. Cell Death Dis, 2016, 7(12): e2524.

[28] AbumareeM, AlJumah M, Pace RA, et al. Immunosuppressive properties of mesenchymal stem cells. Stem Cell Rev, 2012, 8(2): 375-92.

[29] Brunel M, Herr F, Durrbach A. Immunosuppressive properties of mesenchymal stem cell. Curr Transplant Rep, 2016, 3(4): 348-57.

[30] Xü J, Ming Q, Wang X, et al. Mesenchymal stem cells moderate immune response of type 1 diabetes. Cell Tissue Res, 2017, 368(2): 239-48.

[31] Nehlin J, Isa A, Barington T, Immunogenicity and Immune-modulating properties of human ctem cells. In: Ghodamrezanzhad A. Editors. Stem Cells in Clinic and Research. InTech, 2011, 10.5772/17868.

[32] Korn T, Bettelli E, Oukka M, et al. IL-17 and Th17 cells. Annu Rev Immunol, 2009, 27(1): 485-517.

[33] Shi Y, Su J, Roberts AI, et al. How mesenchymal stem cells interact with tissue immune responses. Trends Immunol, 2012, 33(3): 136-43.

[34] Fan L, Hu C, Chen J, et al. Interaction between mesenchymal stem cells and B-cells. Int J Mol Sci, 2016, 17(5): 650.

[35] Freitag J, Bates D, Boyd R, et al. Mesenchymal stem cell therapy in the treatment of osteoarthritis: reparative pathways, safety and efficacy'a review. BMC Musculoskelet Disord, 2016, 17: 230.

(Received 21 December 2017, Revised 23 January 2018, Accepted 30 January 2018) 